\title{
Des membranes qui bullent
}

\author{
Jean-Marc Allain et Martine Ben Amar \\ Laboratoire de Physique Statistique, École Normale Supérieure, Paris
}

\begin{abstract}
Les lipides présents dans la membrane cellulaire s'organisent spontanément dans l'eau en une vésicule, formée d'une double couche de molécules, quasiment plane, repliée en un grand sac. Les vésicules sont des systèmes modèles de la membrane cellulaire. Ce sont aussi des systèmes physiques intéressants, car elles sont à la fois bidimensionnelles et très anisotropes : fluide à l'intérieur d'une couche et élastique perpendiculairement. Dans les vésicules formées d'un mélange de lipides, imitant mieux la composition cellulaire, une séparation de phase peut se produire, avec la formation de domaines, modélisant les "rafts " de la membrane cellulaire. La présence de ces domaines favorise des instabilités, élastiques ou hydrodynamiques. Nous présentons les propriétés des vésicules, particulièrement celles liées à la présence de ces domaines, ainsi que des interprétations physiques de trois instabilités observées expérimentalement.
\end{abstract}

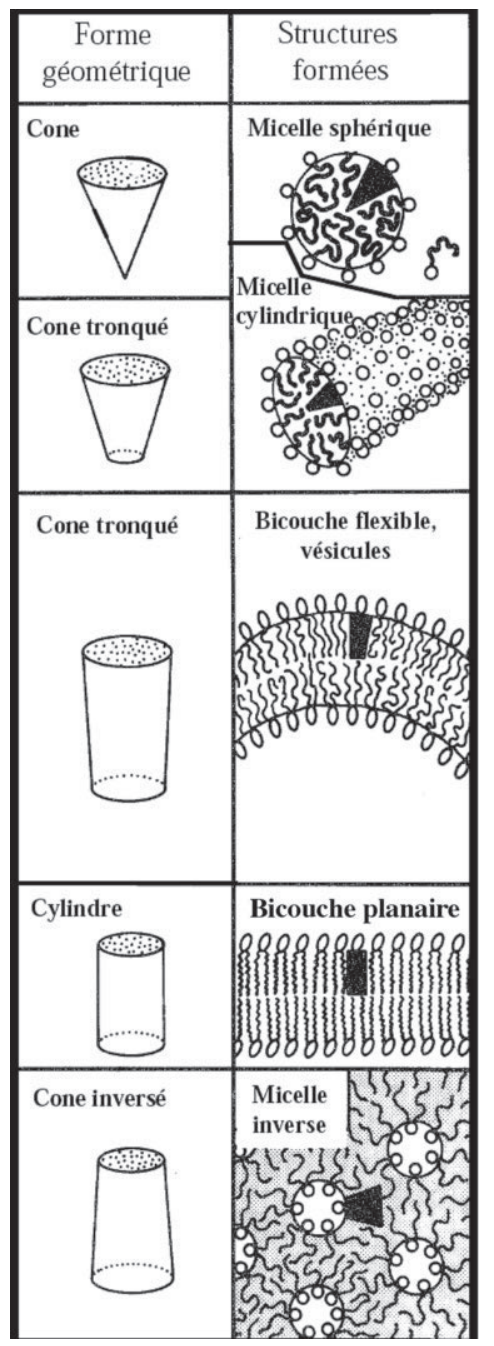

Figure 1: Organisation des molécules amphiphiles en fonction de leur forme géométrique. Les lipides ont une forme cylindrique et s'organisent spontanément en bicouches. Les détergents classiques ont une forme conique et s'organisent en micelles sphériques ou en micelles cylindriques. D'autres formes peuvent exister, comme les micelles inversées pour des molécules amphiphiles, avec une partie hydrophobe plus large que la tête hydrophile. (Figure extraite de la thèse de J.B. Manneville, Université Paris 7, 1999).

Les graisses alimentaires sont composées de trois familles de lipides : les triglycérides, les phospholipides et le cholestérol. Les triglycérides servent principalement de réserve d'énergie, alors que les phospholipides et le cholestérol ont un rôle structurel : ils forment les parois des cellules. En particulier, le taux de cholestérol influe sur les propriétés mécaniques de la paroi. Aussi, comprendre les propriétés physiques des structures de lipides est un enjeu important pour comprendre la mécanique de la membrane cellulaire et donc le fonctionnement des cellules.

Les phospholipides sont des molécules amphiphiles, à l'instar des détergents : ils possèdent une tête hydrophile et une queue hydrophobe. Pour les phospholipides, la queue hydrophobe est formée de deux chaînes carbonées, contre une seule pour les détergents classiques, ce qui donne aux phospholipides une géométrie cylindrique et non conique. C'est cette géométrie qui détermine l'organisation des molécules dans l'eau afin d'écranter au mieux les parties hydrophobes.

Ainsi, des molécules coniques vont s'organiser en micelles, c'est-à-dire des coques dont la face extérieure est formée par les têtes hydrophiles, tandis que les phospholipides vont s'organiser en bicouches, constituées de deux épaisseurs de molécules tête bêche avec les têtes hydrophiles à l'extérieur (figure 1).

Ces bicouches de lipides ont une épaisseur d'environ quatre nanomètres. Dans l'eau, elles se referment sur elles-mêmes, formant des grands sacs appelés vésicules. Le rayon d'une vésicule varie selon les conditions de fabrication, entre cent nanomètres et dix micromètres. Dans la suite, nous nous intéresserons aux vésicules de grands rayons, dites Vésicules Géantes Unilamellaires (VGU), qui présentent l'intérêt d'être observables facilement au microscope.

Les vésicules nous intéressant ici sont utilisées comme système modèle de la membrane cellulaire. Elles sont formées d'un mélange de lipides (phosphatidylcholine, sphyngomyéline et cholestérol), en proportion variable suivant l'expérience. Parmi ces lipides, seul le cholestérol n'est pas un phospholipide, mais un stérol ; sa présence n'empêche toutefois pas (dans les concentrations utilisées) l'organisation des molécules en une bicouche. Ce type de vésicules peut présenter une séparation de phases, conduisant à l'apparition de domaines de compositions différentes dans la membrane. Des structures similaires pourraient aussi exister in vivo et être impliquées dans différents mécanismes tels que le transport à l'intérieur de la cellule, l'adhésion ou la pénétration de virus. Nous avons étudié trois instabilités liées à la présence de domaines lipidiques sur la vésicule. Ceci nous renseigne sur les propriétés mécaniques de la bicouche de lipides en présence de plusieurs phases. Ces travaux permettent de mieux comprendre certains phénomènes physiques, comme les écoulements bidimensionnels, mais aussi comment la présence de domaines peut être mise à profit par la cellule, par exemple pour trier des lipides ou des protéines.

\section{Propriétés mécaniques des vésicules}

La structure de la bicouche impose une orientation aux lipides : les chaînes hydrophobes sont orientées perpendiculairement au plan de la bicouche (figure 1). Les lipides sont confinés dans les feuillets par l'eau environnante qui empêche la sortie de la queue hydrophobe. L'absence d'interaction spécifique forte entre les molécules de lipides leur permet de se déplacer les unes par rapport aux autres dans le plan de la bicouche. Celle-ci est donc un milieu fortement anisotrope, dont la structure est similaire à celle des cristaux liquides de type smectique (dans lesquels les molécules sont parallèles les unes aux autres et regroupées en couches parallèles). À l'inverse des cristaux liquides, les bicouches ont une épaisseur très petite devant les autres dimensions, ce qui en fait un bon exemple de matériau bidimensionnel.

Les propriétés mécaniques de la bicouche sont liées à cette anisotropie. Les déformations qui tendent à écarter les têtes hydrophiles sont défavorisées, car elles exposent le cœur hydrophobe. Les lipides sont très peu solubles dans l'eau environnante, contrairement aux molécules de savons usuels, de forme conique ; aussi, la surface de la vésicule, à l'échelle moléculaire, reste toujours constante. La bicouche se comporte comme un matériau élastique pour les déformations hors de son plan (courbure) : les têtes du feuillet externe sont légèrement écartées et veulent se refermer. Par contre, déplacer les lipides sans écarter les têtes ne demande pratiquement pas d'énergie. Aussi, pour des déformations dans le plan de la bicouche, les lipides se com- 
portent comme un fluide incompressible, l'écoulement des molécules se faisant à surface constante.

Les propriétés élastiques de la bicouche sont décrites par deux coefficients physiques : le module de flambage et le module de Gauss. Le premier donne l'énergie pour courber la bicouche en dehors de son plan. Le module de Gauss donne l'énergie pour une déformation à courbure moyenne constante (par exemple, pour passer d'une surface plane à une selle de cheval) ; sa contribution est limitée aux changements de topologie ou, dans le cas des vésicules à plusieurs domaines, à une contribution au niveau de l'interface ; aussi, il est délicat à mesurer et mal connu. Les propriétés élastiques peuvent, de plus, prendre en compte une possible dissymétrie entre les deux feuillets, à travers une courbure spontanée. Ce terme n'est pas nécessaire pour les vésicules à plusieurs domaines, car leur membrane est symétrique.

L'énergie élastique nécessaire pour courber la bicouche est typiquement de quelques dizaines de fois l'énergie d'agitation thermique. Aussi, celle-ci est suffisante pour provoquer des déformations locales perpendiculaires à la surface de la vésicule, ce qui entraîne des fluctuations de la bicouche autour de sa position d'équilibre. La plupart de ces fluctuations ont lieu à des échelles inférieures à la résolution des microscopes optiques, utilisée comme échelle d'observation, et sont donc invisibles. Elles constituent un réservoir de surface : à l'échelle d'observation, il est possible de puiser dans ces fluctuations invisibles pour augmenter la surface visible de la vésicule. Toutefois, gêner ces fluctuations, en étirant la vésicule ou en approchant un objet, est défavorable car cela diminue l'entropie du système. Ainsi, lorsque l'on approche un objet, il apparaît une force répulsive dite d'Helfrich, qui empêche l'adhésion spontanée. Augmenter la surface (visible) de la vésicule est possible, mais avec un coût énergétique similaire à une tension de surface. Cette tension effective dépend des fluctuations supprimées et donc de l'étirement de la vésicule : plus la vésicule est tendue, plus il est difficile de l'étirer.

La bicouche est aussi un milieu semi-perméable : les grosses molécules ou les ions ne peuvent pas la traverser, mais elle ne bloque pas complètement les molécules d'eau. Une pression osmotique existe donc entre l'intérieur et l'extérieur de la vésicule, si les concentrations en grosses molécules ou en ions ne sont pas les mêmes à l'intérieur et à l'extérieur. Modifier cette pression permet de gonfler ou non la vésicule et, ainsi, de modifier sa forme et sa tension de surface. Dans la suite, nous considérerons des VGU tendues, c'est-à-dire suffisamment gonflées pour qu'il n'y ait plus de fluctuations visibles en microscopie optique.

Les vésicules sont donc des milieux complexes qui possèdent des propriétés à la fois des bulles de savon, contrôlées par la tension de surface, des milieux élastiques et des liquides [1]. La plupart de ces propriétés se retrouvent dans les parois cellulaires. Par exemple, les chocs osmotiques (c'est-à-dire des changements rapides de la pression osmotique) sont couramment utilisés par les biologistes pour gonfler la cellule, puis faire exploser la paroi et ainsi récupérer le contenu cellulaire. Les propriétés mécaniques des cellules sont toutefois encore plus complexes: la cellule possède une architecture intérieure qui maintient sa forme et contrôle les fluctuations, modifiant le rôle de la tension de surface.

\section{Vésicules inhomogènes}

Les parois cellulaires sont formées d'un mélange de lipides, ce qui peut être reproduit dans les VGU. Lorsqu'une vésicule est formée de plusieurs lipides différents, une séparation de phase peut avoir lieu, conduisant à la formation de domaines de compositions différentes (figure 2).

Les domaines constitués de cholestérol et de sphingomyéline font actuellement l'objet de nombreuses études. Ces domaines apparaissent dans des vésicules formées d'un mélange de phosphatidylcholines (que, pour simplifier, nous dénommerons désormais phospholipides), de sphingomyélines et de cholestérols ; ils sont enrichis, par rapport à la bicouche environnante, en choles-

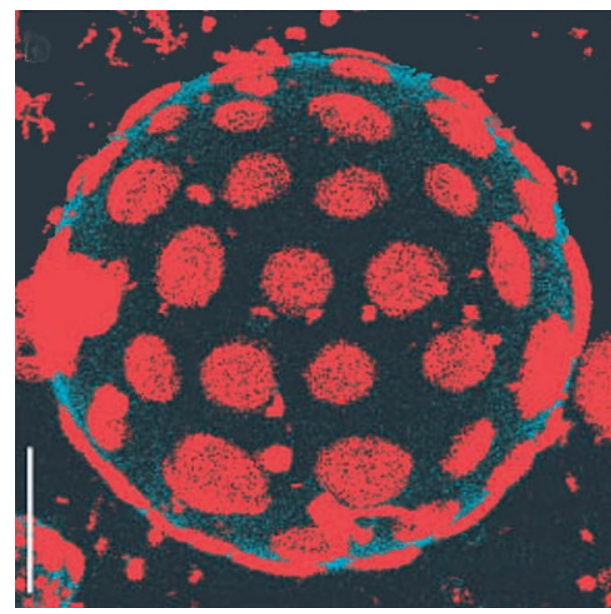

térol et sphingomyéline et appauvris en phospholipides. La séparation de phase vient d'une interaction attractive, probablement due à des liaisons hydrogènes, entre les molécules de cholestérol et de sphingomyéline. Aussi, ces domaines n'apparaissent que pour une gamme de composition donFigure 2: Vue en microscopie de fluorescence d'une née et pour des vésicule comportant deux phases différentes. La températures en phase classique "liquide-désordonnée " est formée dessous d'une

d'îlots marqués en rouge et la phase "liquide-ordon- valeur critique. La née » est le domaine connexe marqué en bleu. Barre d'échelle : $5 \mu$ m. (Image extraite de [2] avec l'autor'sation des auteurs et de l'éditeur.) présence des molécules de cholestérol gêne les mouvements des molécules de sphingomyéline, mais pas au point de supprimer la fluidité de la membrane ; aussi, les domaines enrichis en cholestérol et sphingomyéline sont dits " liquides-ordonnés » et conservent les mêmes propriétés mécaniques qu'une bicouche classique, dite "liquide-désordonnée ", mais avec des coefficients physiques différents : la viscosité du domaine "liquide-ordonné " est supérieure à celle du domaine "liquide-désordonné ", de même que sa rigidité de courbure. De plus, ils sont légèrement plus épais que la bicouche classique environnante, cinq nanomètres au lieu de quatre. L'épaisseur de l'interface entre deux domaines est petite par rapport à la taille des domaines et peut donc être vue comme une ligne sur la surface. Cette ligne est équivalente sur une surface bidimensionnelle de l'interface dans un mélange eau-huile ; l'existence de cette interface a un coût, donné par une tension de ligne équivalente à deux dimensions de la tension de surface dans un mélange de liquides usuels. Cette tension de ligne est homogène à une force et sa valeur est de l'ordre de $0,5 \mathrm{pN}$.

Ce type de domaines pourrait reproduire, à des tailles nettement plus grandes, des domaines similaires, appelés rafts, qui pourraient exister dans la cellule. Bien qu'il n'y ait pas actuellement d'observation directe in vivo des rafts, ils semblent être impliqués dans de nombreux processus cellulaires, comme le transport des lipides ou des protéines, ou la signalisation membranaire [3].

Dans la suite, nous présentons trois exemples d'instabilités de forme de membranes hétérogènes. Les deux premiers exemples illustrent les influences combinées de la tension de surface, de l'élasticité et de la tension de ligne, avec des déformations perpendiculaires à la surface de la vésicule, allant dans les deux cas jusqu'à l'éjection des domaines, qui forment de nouvelles vésicules. Le troisième exemple illustre un changement de la forme des domaines liquides-ordonnés dû à des effets hydrodynamiques. Dans les trois cas, ces phénomènes peuvent être compris par des analyses de stabilité, qui permettent une bonne description des expériences. À chaque fois, l'instabilité est liée à la présence de domaines liquides-ordonnés.

\section{Rupture de tubes membranaires}

Une des étapes clés du transport intra-cellulaire est la fission de la membrane, qui transforme une vésicule en deux. Un mécanisme possible est basé sur la formation puis la rupture de tubes de membrane de rayon nanométrique : après la rupture du tube, une partie de la bicouche ne sera plus connectée à la vésicule 


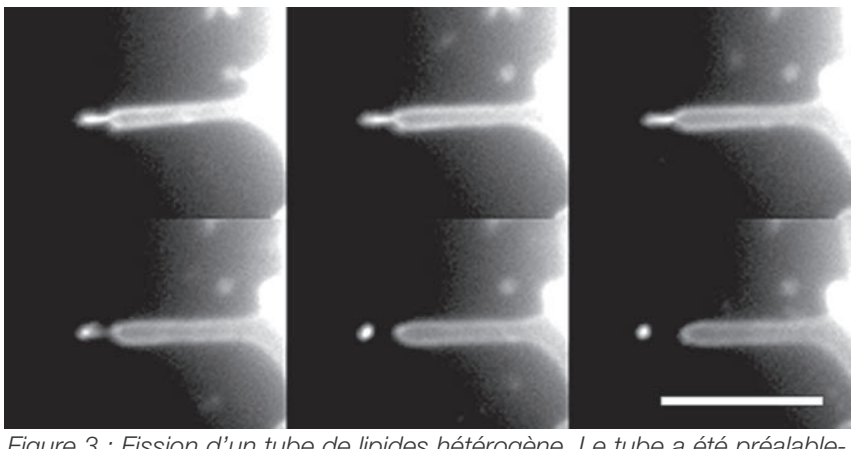

Figure 3 : Fission d'un tube de lipides hétérogène. Le tube a été préalablement formé à l'aide de moteurs moléculaires (kynésines) marchant sur un microtubule. La force est exercée par une accumulation de moteurs à la pointe, mais des moteurs se trouvent sur toute la longueur du tube. La partie brillante et fine initialement à la pointe du tube est un domaine "liquidedésordonné » riche en DOPC. La partie large est un domaine "liquideordonné ". La fission se produit aux sites de formation des petits domaines résultant de la séparation de phase. Le temps entre deux images est d'une seconde. Barre d'échelle : $10 \mu \mathrm{m}$. (Image extraite de [5]. Cette expérience a été réalisée à l'Institut Curie à Paris.)

mère et formera une nouvelle vésicule. Un tube peut être formé en tirant sur une vésicule. La formation du tube est liée à une combinaison entre l'élasticité de la bicouche et sa tension de surface. Lorsqu'une force de traction est appliquée sur une vésicule faiblement courbée, celle-ci se déforme d'abord sous l'action de la tension de surface, avec une forme en citron. Toutefois, la bicouche est fortement courbée au niveau des points d'application de la force, ce qui a un coût élastique important. Pour éviter la divergence de l'énergie élastique à la pointe, il est préférable, au-delà d'une force critique, de former un tube [4] : l'énergie est ainsi stockée sur toute la longueur du tube et la forme de l'extrémité reste fixe. Notons que la formation d'un tube demande à la fois de courber la membrane en un cylindre, ce qui a un coût élastique, et de prélever de la surface dans la vésicule-mère, ce qui a un coût lié à la tension de surface de celle-ci. Le rayon du tube est alors donné par un équilibre entre les énergies de courbure et de tension de surface, la pression osmotique n'ayant qu'un rôle très faible. Les tubes ainsi formés ont un rayon typique entre 20 et $100 \mathrm{~nm}$. Une particularité des tubes de membrane est que, une fois le tube initié, la force exercée est suffisante pour le faire croître sur de grandes longueurs, car il puise sa surface dans les réserves de fluctuations de la vésicule.

À notre connaissance, il n'existe pas d'expériences montrant l'extraction de tubes de membrane à plusieurs domaines. Toutefois, il est possible de créer un tube à plusieurs domaines en induisant la séparation de phase une fois le tube formé. La formation des domaines peut être obtenue en modifiant légèrement la composition, par exemple en prélevant des molécules de cholestérol par une oxydation photo-induite, dans une membrane proche de la séparation de phase. Une fois la séparation induite, le tube se rompt au niveau de l'interface en typiquement une seconde (figure 3 ).

Pour comprendre ce mécanisme de rupture, nous avons simulé un système formé de deux domaines tubulaires semi-infinis. La forme du tube est complètement donnée par trois paramètres la tension de ligne de l'interface entre les deux domaines, l'écart entre leurs modules de flambage, ainsi que celui de leurs modules de Gauss. Ainsi, il est possible de déterminer la forme du tube, en particulier au niveau de l'interface, mais il s'agit de détails trop petits pour être observés expérimentalement. Aussi, nous avons calculé une grandeur accessible, le temps de fission, en utilisant un modèle de processus activé. Un ordre de grandeur de la barrière d'énergie est donné par l'écart d'énergie entre la forme calculée du tube connecté et la forme infiniment pincée, juste avant séparation. Le temps caractéristique est celui du mouvement de l'eau nécessaire pour pincer le tube. Nous obtenons alors un temps de fission de l'ordre de $500 \mathrm{~ms}$. Cet ordre de grandeur est en bon accord avec les mesures expérimentales, autour d'une seconde. L'étude des tubes à plusieurs domaines illustre l'importance des propriétés élastiques sur le devenir de la bicouche. De plus, elle montre la fragilité des systèmes à deux domaines comparée à celle de systèmes homogènes.

\section{Éjection de domaines par absorption de molécules}

II est aussi possible d'obtenir expérimentalement la fission d'une vésicule à deux domaines, par un ajout de molécules extérieures. L'ajout de protéines comme la PLA2 ou de détergents qui vont venir s'absorber à la surface de la membrane va provoquer une déformation de la vésicule, avec un "soulèvement » du domaine liquide-ordonné. Les protéines ou les détergents forcent en effet la membrane à se courber localement, en raison, par exemple, de leurs formes coniques (figure 1). Ceci se traduit par un couplage entre la courbure de la membrane et la concentration en molécules. Si suffisamment de molécules sont absorbées sur la surface, la déformation va jusqu'à la séparation des deux domaines en deux vésicules distinctes (figure 4).

Une membrane tendue se comporte essentiellement comme une bulle de savon, sauf à proximité de la jonction entre les deux domaines, où l'énergie élastique est non négligeable. La vésicule est alors constituée de deux calottes, connectées par une mince bande élastique. On obtient une situation similaire à celle d'un pli sur une feuille de papier, où est localisée l'énergie élastique. Cette approche simplifie le formalisme, ce qui permet une étude analytique du système. Deux instabilités différentes peuvent avoir lieu, concernant soit une des calottes, soit l'ensemble du système.

La première instabilité se produit pour des fortes valeurs du paramètre de couplage ou des concentrations en molécules élevées. La courbure imposée par les molécules est alors suffisante pour lutter avec les forces de pression osmotique, au moins dans l'une des calottes sphériques. Celle-ci n'est alors plus une solution stable et le domaine prend une forme plissée. Cette instabilité s'observe expérimentalement lors de l'ajout de grandes quantités de détergents. Cette instabilité ne se produit que sur une seule des calottes, car les molécules extérieures ne s'absorbent pas de manière identique dans les deux phases.

La deuxième instabilité concerne l'ensemble des deux calottes. Elle peut se produire en parallèle avec la première ou indépendamment. Nous nous sommes plus particulièrement intéressés au cas où le couplage et la concentration en molécules ne sont pas suffisants pour lutter contre la pression osmotique : la forme de la vésicule reste donnée par deux calottes sphériques, ce qui permet un traitement analytique. L'effet des molécules se fait sentir au niveau du pli entre les deux domaines et provoque une aug-
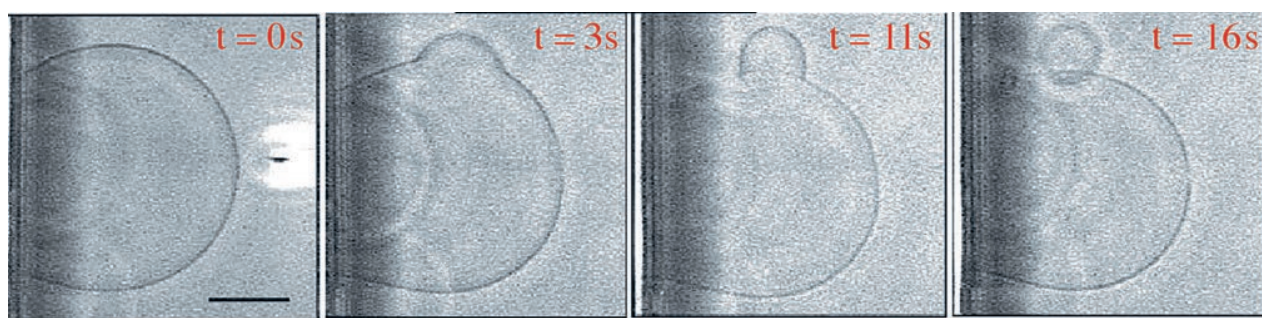

Figure 4 : Éjection d'un domaine liquide-ordonné induite par l'absorption de protéines PLA2, vue en microscopie optique confocale : la vésicule apparaît comme un trait noir dans le plan focal. Les deux domaines ne peuvent pas être distingués ainsi, mais peuvent l'être en fluorescence. L'image de gauche montre la vésicule juste avant l'injection de protéines. La partie gauche de la vésicule est tronquée : la vésicule est connectée à son électrode de croissance. Après l'injection des protéines, le domaine liquide-ordonné commence à se soulever Le processus se poursuit jusqu'à l'éjection complète. Barre d'échelle : $20 \mu \mathrm{m}$. (Ces expériences ont été réalisées au Laboratoire de Physicochimie Biomoléculaire et Cellulaire à Paris. Image extraite de [6] avec l'autorisation de l'éditeur.) 
mentation de la tension de ligne. Ceci provoque une diminution du rayon du pli entre les deux domaines. On observe alors un soulèvement du domaine liquideordonné qui peut aller jusqu'à la fission si la tension de ligne effective dépasse une valeur critique.

Nous pourrions penser que ce mécanisme fait diminuer la taille du raccord entre les deux phases jusqu'à ce qu'il n'y ait plus qu'un cou infiniment fin. Ce n'est pas le cas. Pour une vésicule tendue à deux domaines, il existe, pour une tension de ligne donnée, soit deux solutions, soit aucune. Lorsqu'il existe deux solutions, l'une des deux est instable et ne peut pas être observée expérimentalement. Pour une tension de ligne critique $\sigma_{c}$, les deux solutions se rejoignent et, pour des tensions supérieures, il n'y a plus de solution (figure 5).
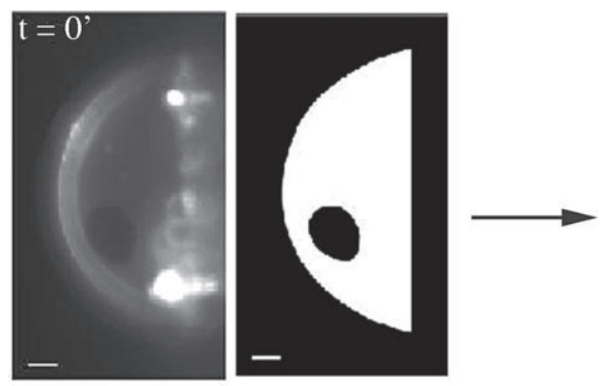

L

Figure 6: Instabilité de succion dans une vésicule comportant un domaine liquide-ordonné. Les images sont faites en microscopie de fluorescence, le domaine apparaissant comme un cercle noir ; pour une plus grande visibilité, l'image a été mise en noir et blanc à côté. Le domaine est initialement circulaire. En présence de protéines prélevant le cholestérol de la membrane, sa surface diminue et, si cette succion est suffisante, son contour cesse d'être circulaire et présente des ondulations. (Les expériences ont été réalisées par A. Lamazière,

Au moment de la fission, le rayon du pli n'est donc pas nul, mais il n'y a plus de solutions connectées possibles et le système rompt.

Cette instabilité est similaire à celle d'un film de savon tendu entre deux anneaux : lorsque les anneaux sont proches, il existe deux solutions en forme de caténoïdes ${ }^{1}$, une stable et une instable. Lorsque l'écartement des anneaux augmente, les deux caténoïdes se rapprochent et, lorsqu'elles sont suffisamment proches, le système devient instable. II évolue irréversiblement alors vers deux disques de savon portés par les anneaux.

\section{Instabilité de succion}

Les domaines liquides-ordonnés permettent aussi de sonder les propriétés hydrodynamiques de la membrane. Des travaux récents, à l'hôpital Saint-Antoine à Paris, ont montré que des constituants du sang pouvaient pomper la phase liquide-ordonnée en lui enlevant du cholestérol (figure 6).

Suivant les conditions expérimentales, la surface du domaine liquide-ordonné diminue, soit en restant circulaire, soit avec des

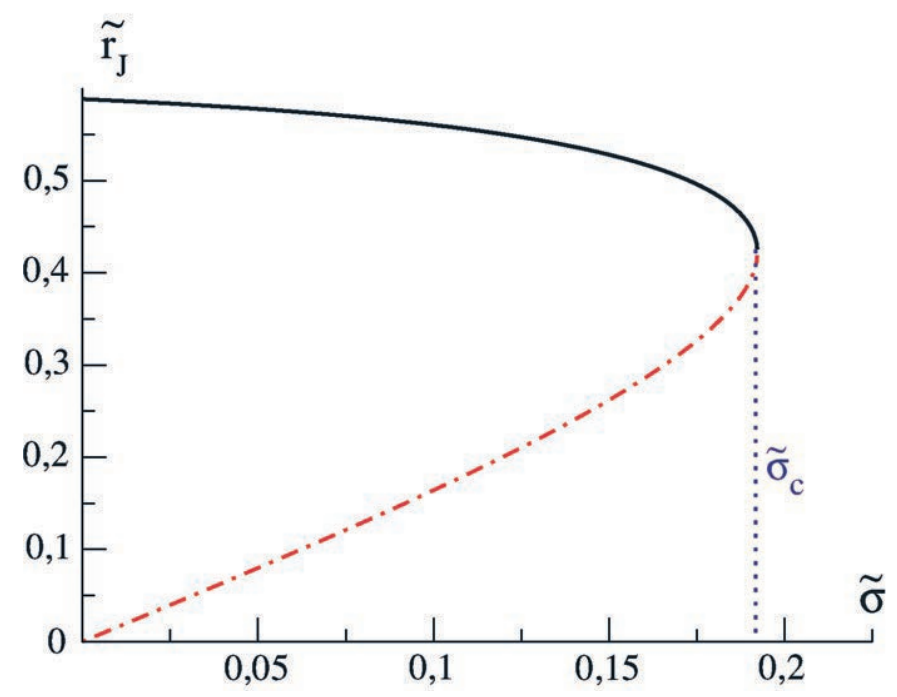

Figure 5 : Longueur de l'interface entre les deux domaines en fonction de la tension de ligne effective pour une vésicule à deux domaines tendue. En dessous d'une valeur critique, deux solutions existent : une stable (courbe noire) et une instable (courbe rouge). Au-delà de la valeur critique, il n'y a plus de solution connectée et les deux domaines se séparent irréversiblement. Le calcul a été fait avec 9,5\% de la surface occupée par le domaine liquide-ordonné. Les grandeurs sont sans dimension. L'échelle de longueur est donnée par le rayon de la sphère dont la surface est égale à celle de la vésicule ; l'échelle de tension de ligne est donnée par la pression osmotique multipliée par l'échelle de longueur au carré.

1. La caténoïde est la surface de révolution, solution de l'équation $r(z)=R \cosh (z / R)$ en coordonnées cylindriques. oscillations du contour. Cette instabilité du bord rappelle celle de Saffman-Taylor, qui se produit lorsqu'un fluide visqueux, confiné entre deux plaques très proches, est chassé par un fluide peu visqueux.

L'instabilité du bord est due à la succion vers le centre du domaine : celle-ci provoque un écoulement de plus en plus rapide des lipides vers le centre. Un point du contour du domaine proche du centre va donc être plus accéléré qu'un point plus loin du centre, favorisant le développement d'une instabilité. La tension de l'interface s'oppose à l'instabilité, car elle veut réduire la longueur du bord. Ce système favorise des instabilités à grandes longueurs d'onde, comme observé dans l'expérience. Si la succion n'avait pas lieu principalement dans la phase liquide-ordonnée, l'instabilité n'aurait pas lieu et le domaine resterait circulaire.

Les résultats sont très différents de l'instabilité de SaffmanTaylor, pour laquelle le moteur est la différence de viscosité entre les deux fluides, qui n'intervient pas pour les bicouches. D'autre part, l'instabilité de Saffman-Taylor tend plutôt à favoriser des instabilités avec un grand nombre initial d'oscillations. Nous illustrons par cette étude la différence entre un liquide subissant un écoulement confiné et un fluide bidimensionnel plongé dans un réservoir. Cette étude permet aussi de prédire un taux de succion minimal pour que l'instabilité de contour se développe, ce qui correspond aux observations expérimentales.

\section{Conclusion}

L'étude des instabilités mécaniques ou hydrodynamiques apporte des informations intéressantes sur le comportement des membranes lipidiques, des structures possédant des caractéristiques bidimensionnelles. En particulier, la présence de domaines plus structurés, mimant des structures cellulaires, permet de noumême si le système reste simple à côté d'une cellule. De plus, ces domaines membranaires rendent les vésicules plus sensibles aux perturbations extérieures et, ainsi, permettent de sonder l'ensemble des propriétés physiques de la membrane : son élasticité, sa tension de surface et sa fluidité. Ces instabilités, spécifiques de domaines, peuvent être impliquées dans le fonctionnement de la cellule, par exemple pour créer une différence de composition lipidique entre les différents compartiments ou pour permettre la pénétration d'un virus.

\section{Références}

[1] U. Seifert, Advances in Physics, 46, 13 (1997)

[2] T. Baumgart, S.T. Hess, W.W. Webb, Nature, 425, 821 (2003)

[3] K. Farsad, P. De Camilli, Current Opinion in Cell Biology, 15, 372 (2003)

[4] T.R. Powers, G. Huber, R. E. Goldstein, Phys. Rev. E, 65, 041901 (2002) ;

I. Derenyi, F. Julicher, J. Prost, Phys. Rev. Lett., 88, 238101 (2002)

[5] J.M. Allain, C. Storm, A. Roux, M. Ben Amar, J.F. Joanny, Phys. Rev. Lett., 93, 158101 (2004)

[6] G. Staneva, M. I. Angelova, K. Koumanov, Chem. Phys. of Lipids, 129, 53 (2004) velles instabilités, dont l'étude est biologiquement pertinente, 